\title{
Novel sporozoite-based ELISpot assay to assess frequency of parasite-specific B cells after vaccination with irradiated sporozoites
}

\author{
Tanmaya Atre ${ }^{1}$, Tanisha M. Robinson ${ }^{1}$, Tatyana Savransky² ${ }^{2}$, Sheetij Dutta ${ }^{1}$, Judith E. Epstein ${ }^{3}$ \\ and Elke S. Bergmann-Leitner ${ }^{1 *}$ (D)
}

\begin{abstract}
Background: Whole parasite vaccination is an efficacious strategy to induce sterile immunity and to prevent malaria transmission. Understanding the mechanism and response of immune cells to vaccines plays a critical role in deciphering correlates of protection against infection and disease. Immunoassays, such as ELISpot, are commonly used to assess the immunogenicity of vaccines towards $T$ cells and B cells. To date, these assays only analyse responses to specific antigens since they are based on recombinant parasite-derived proteins or peptides. There is the need for an agnostic approach that allows the evaluation of all sporozoite-associated antigens.
\end{abstract}

Methods: ELISpot plates coated with a defined amount of lysed Plasmodium falciparum sporozoites were used to assess the frequency of sporozoite-specific B cells in peripheral blood mononuclear cells from donors immunized with either a recombinant malaria vaccine or irradiated sporozoites.

Results: This report describes the assay conditions for a specific and sensitive sporozoite-based B cell ELISpot assay. The assay development considers the quality of sporozoite preparation as well as the detection threshold of the frequency of antigen-specific B cells. The assay enables the detection of sporozoite-specific lgM and lgG-producing $B$ cells. Moreover, the assay can detect sporozoite-reactive B cells from subjects that were either vaccinated with the radiation attenuated sporozoite vaccine or a recombinant pre-erythrocytic vaccine.

Conclusion: The newly developed sporozoite-based B cell ELISpot enables the monitoring of changes in the frequency of sporozoite-specific B cells. Applying this assay to assess the potency of vaccination regimens or seasonal changes in B cell populations from subjects residing in malaria-endemic areas will provide an opportunity to gain insight into immune mechanisms involved in protection and/or disease.

Keywords: Malaria, Sporozoite, Whole parasite vaccine, ELISpot, B cells, Antibodies, Immunity

\section{Background}

Malaria parasites start their mammalian life cycle upon injection via mosquito bite into the host (reviewed in [1, 2]). Sporozoites migrate in the skin and most of them eventually either enter the lymphatic system or the blood stream by crossing the vascular endothelium. The sporozoites will arrive in the liver, the target site for sporozoite

\footnotetext{
*Correspondence: elke.s.bergmann-leitner.civ@mail.mil

${ }^{1}$ Malaria Vaccine Branch, US Military Malaria Research Program, Walter

Reed Army Institute of Research, Silver Spring, MD, USA

Full list of author information is available at the end of the article
}

infection. Within the liver, a high replication of parasites occurs, where one infected hepatocyte will ultimately release up to 30,000 merozoites. This starts the erythrocytic stage of the infection, which is responsible for morbidity and, in case of Plasmodium falciparum, often mortality. Some of the erythrocytic parasites develop into sexual forms (gametocytes), which are ultimately picked up by mosquitoes during a blood meal thus completing the transmission cycle.

Recently, proteomic profiling revealed that P. falciparum sporozoites express 1991 proteins [3]. Current vaccine models include targeting surface proteins of the 
sporozoite laying the groundwork for sub-unit vaccines. Circumsporozoite protein (CSP) is the most studied vaccine target since it is the most abundantly expressed surface antigen on the sporozoite. The most promising sub-unit vaccine is the RTS,S/AS01 vaccine, which is a fusion of the hepatitis surface antigen (HBsAg) as carrier with the central repeat region and the $\mathrm{C}$-terminus of CSP. This vaccine has achieved $50-86 \%$ protection against experimental sporozoite challenge depending on the vaccination regimen $[4,5]$. Whole parasite vaccines (WPV) are attractive as they have the potential to induce immune responses against a wide range of antigens associated with the sporozoite. Attenuation is achieved through radiation attenuated (RAS) or genetically engineered sporozoites (GAS) or immunizing with non-attenuated parasites while treating vaccinees with anti-malarial drugs (reviewed in [6-8]). The oldest approach, the RAS vaccine, initially employed delivery through bites by irradiated mosquitoes and exposing study participants to a large number of bites in monthly intervals [9]. Attenuated sporozoites are able to infect the liver, but depending on the attenuation protocol, the parasites stall their development into infectious blood-stage parasites. Immunization with RAS leads to sterile protection that breaks the transmission cycle and is, therefore, considered the gold standard for developing recombinant or sub-unit vaccines.

The RAS vaccine has been reported to mediate protection through an array of immune mechanisms; antibodies, $\mathrm{CD}^{+}{ }^{+}, \mathrm{CD}^{+}{ }^{+}$and $\gamma \delta \mathrm{TCR} \mathrm{T}$ cells have all been shown to play a crucial role in protection $[10,11]$. While there is a strong focus on the identification of cellular mechanisms, the characterization of sporozoite-specific B cells has been unable to garner similar enthusiasm even though preclinical models have identified a crucial role for B cells in parasite clearance $[12,13]$. In humans, one study describes the induction of B cells after GAS vaccination [14]. Here, the B cells were tested in ELISpot assays with nine plasmodial antigens (of which four are expressed on the sporozoite). No comparable analysis has been reported so far with RAS-induced B cells.

$\mathrm{B}$ cell ELISpots are a relatively simple and robust method to measure the frequency of antibody-secreting memory B cells in peripheral blood mononuclear cells. ELISpot assays enable the quantification of antigenspecific B cells and can be considered complimentary to ELISA assays. While serum ELISA assays determine the titres of antigen-specific antibodies, they do not provide information about the number of antigen-specific $B$ cells. Combining ELISA results with ELISpot data provides deeper insights into humoral immunity to vaccines. Recently, the development of a sporozoite-based ELISA method was reported [11]. It allows the detection of sporozoite-specific antibodies that a classic ELISA based on recombinant proteins or peptides failed to report. Similarly, the present study overcomes the need for recombinant proteins or peptides when measuring antigen-specific B cells that are specific for this particular antigen. In contrast, the method offers the opportunity to agnostically evaluate and quantify all sporozoite-specific B cells by using either intact or lysed sporozoites as plate antigens. Subsequent integration of ELISpot and ELISA data from these sporozoite-based assays will support an in-depth analysis of the frequency of vaccine induced, parasite specific B cell responses and the type of antibodies they secrete. The sensitivity of the assay makes it possible to perform the analysis even when only a limited cell number is available.

\section{Methods}

Parasite strain and reagents

Plasmodium falciparum 3D7 strain was obtained from the insectary at the Walter Reed Army Institute of Research. Recombinant, full length $P$. falciparum CSP (3D7) was produced in Escherichia coli as previously described [15].

\section{Sporozoite dissection}

Sporozoites (SPZ) were prepared by dissecting mosquitoes 16-20 days post blood feed using the Ozaki method [16], or the clean dissection method. The Ozaki method is a fast and simple dissection of mosquito heads followed by centrifugation of the material through a glass wool pillow; for the clean dissection method [17], salivary glands are removed from the mosquito heads, pooled and then disrupted by shear forces using a syringe and $18 \mathrm{~g}$ needle. SPZ were frozen as pellets and stored at $-80^{\circ} \mathrm{C}$ to be used as lysates. Upon thawing, pellets were resuspended in PBS ( $\mathrm{pH} 7.2$ ), vigorously pipetted using siliconized/ pre-lubricated pipette tips (to minimize loss of material), and lysis confirmed by microscopy.

\section{Memory B cell stimulation}

Peripheral blood mononuclear cells (PBMC) from previously conducted clinical trials [IMRAS; Immunization by mosquito bite with radiation attenuated SPZ (NCT01994525) and MAL027 (NCT00075049)] were used. In the IMRAS trial, study participants received 5 doses of approximately 200 infectious bites/dose by $P$. falciparum-infected Anopheles stephensi mosquitoes. The goal of the trial was, apart from safety and immunogenicity, to develop reagents for the identification and validation of biomarkers of protection (Epstein et al. pers. commun.). In the MAL27 trial, study participants were immunized with three doses of RTS,S adjuvanted with AS01B or AS02A [18]. Cells from the MAL27 trial were 
used to represent 'CSP-immune' individuals. PBMC were thawed and counted using a Luna- $\mathrm{FL}^{\mathrm{TM}}$ Dual Fluorescence cell counter (fluorescence protocol with AO/PI to determine cell viability). Warm culture medium [(RPMI1640 containing $10 \%$ fetal bovine serum (FBS), Pen/ Strep, L-glutamine, non-essential amino acids (NEAA), Sodium Pyruvate, 2-mercaptoethanol] was prepared. Cell numbers were adjusted to $1.5 \times 10^{6}$ cells $/ \mathrm{ml}$ and stimulated with $1 \mu \mathrm{g} / \mathrm{ml}$ of the TLR7/8 ligand R848 (Mabtech) and $10 \mathrm{ng} / \mathrm{ml}$ recombinant human (rh) IL-2 (Mabtech) in culture medium for 36 to $48 \mathrm{~h}$ at $37{ }^{\circ} \mathrm{C}$ in a humidified incubator with $5 \% \mathrm{CO}_{2}$ in six-well plates (Corning, Tewksbury, MA) Stimulating B cells polyclonally with R848 and supporting full activation with recombinant IL-2 ensures that all B cell clones are activated and produce immunoglobulins. Sporozoite material on the ELISpot plates enabled the capturing of malaria-specific antibodies secreted while incubating the cells in the ELISpot plates.

\section{Sporozoite-specific lgM/lgG ELISpot assay}

PVDF-based membrane plates (MSIP, Millipore, Burlington, MA, USA) were activated with $15 \mu \mathrm{l} /$ well $35 \%$ ethanol for $1 \mathrm{~min}$ following the manufacturer's instructions. The plates were washed five times with tissue culture grade, sterile water. Next, the activated ELISpot plates were coated with $100 \mu \mathrm{l} /$ well that equate to 30,000 lysed $\mathrm{SPZ} /$ well or recombinant CSP protein $(1 \mu \mathrm{g} / \mathrm{ml})$. The optimal amount of SPZ was determined in a previous study [11]. Assay controls were: (a) Bovine Serum Albumin (BSA, cell culture grade, Sigma Aldrich, St. Louis, $\mathrm{MO}$, USA, $1 \mu \mathrm{g} / \mathrm{ml}$ ) as the negative control to determine non-specific binding of antibodies to plates; (b) IgM-specific monoclonal antibody (mAb) MT11/12 (Mabtech) or IgG specific mAb MT91/145 (both at $15 \mu \mathrm{g} / \mathrm{ml}$; Mabtech) as positive control since they capture any secreted antibody regardless of specificity. Plates were incubated overnight at $4{ }^{\circ} \mathrm{C}$ [11]. Liquid from wells coated with sporozoite lysate was gently removed and wells allowed to air dry since immobilizing the sporozoite material by air drying rather than fixation is superior regarding the reactivity with antibodies [11]. On the day of the experiment, plates were blocked with culture medium (RPMI 1640 with $10 \%$ FBS and supplements) for $2 \mathrm{~h}$ at $37^{\circ} \mathrm{C}$. It was necessary to use FBS in the culture medium since human $A B$ pooled serum caused significant background issues. Next, R848-activated PBMC or CD19 enriched B cells were plated onto the ELISpot plates. The cell concentration of PBMC was $5 \times 10^{5}$ cells/well (the maximum cell number recommended by the manufacturer of the ELISpot reagents) while the cell concentration of enriched B cells was $5 \times 10^{4}$ to $10^{5}$. Cells were plated in triplicates. For B cell enrichment, R848-activated PBMC were used as starting material for the magnetic enrichment of B cells using CD19-microbeads (Miltenyi Biotec, Auburn, CA, USA) following the manufacturer's instructions. In brief, polyclonally activated PBMC were harvested and cell numbers determined using a Luna- $\mathrm{FL}^{\mathrm{TM}}$ cell counter. Cells were pelleted (centrifugation at $300 \mathrm{~g}$ for $10 \mathrm{~min}$ ) and resuspended in degassed MACS buffer [PBS (pH 7.2) with $0.5 \%$ BSA and 2 mM EDTA] at a concentration of $10^{7}$ cells $/ 100 \mu$ l. Twenty $\mu$ l of anti-CD19 microbead-conjugated $\mathrm{mAb}$ was added and incubated for 15 min at $4{ }^{\circ} \mathrm{C}$. Two ml of MACS buffer was then added and cells spun down to remove unbound antibody (300 g, $10 \mathrm{~min}$ ). Finally, cells were resuspended in $500 \mu \mathrm{l}$ MACS buffer and passed through MS columns (Miltenyi Biotec) inserted into an OctoMACS magnet (Miltenyi Biotec). Enriched cells were counted (Luna-FL ${ }^{\mathrm{TM}}$ ) and plated at a cell concentration of $5 \times 10^{4}$ to $10^{5}$ cells/well. Plates were incubated for $24 \mathrm{~h}$ at $37{ }^{\circ} \mathrm{C}$ in a humidified incubator with $5 \% \mathrm{CO}_{2}$ to allow for the capturing of sporozoitespecific antibodies. Cells were removed from the plates by washing (Biotek EL405 plate washer, Biotek Winooski, VT, USA) plates five times with $250 \mu \mathrm{l} /$ well PBS. Detection of spots was achieved by adding biotinylated $\mathrm{mAb}$ MT22 (for IgM) or mAb MT78/145 (for IgG) at $1 \mu \mathrm{g} /$ $\mathrm{ml}(100 \mu \mathrm{l} /$ well; PBS with $0.5 \%$ FBS as diluent) for $2 \mathrm{~h}$ at room temperature. Plates were washed and incubated with alkaline phosphatase-conjugated Streptavidin-alkaline (1:1,000, PBS with $0.5 \%$ FBS as diluent). All reagents were obtained from Mabtech. After $1 \mathrm{~h}$ at room temperature, plates were washed and BCIP/NBT substrate added until spots were visible. Reaction was stopped by washing plates with tap water. Dried ELISpot plates were analysed using the AID Autoimmun Diagnostika GmbH ELISpot reader (Strassberg, Germany) and accompanying software.

\section{Statistical analysis}

Statistically significant differences between the various assay conditions were determined by using two-sided $\mathrm{T}$ tests (Minitab 17, State College, PA, USA).

\section{Results and discussion \\ CSP-specific B cells recognize sporozoites immobilized on ELISpot plates}

A recent report described optimal assay conditions for a sporozoite-based ELISA [11]. In that study, monoclonal antibodies as well as sera from RAS-immune subjects recognized both, sporozoite lysates and intact SPZ. The results from that study demonstrated that, for convenience, sporozoite lysates can be used in lieu of freshly isolated SPZ. In the present study, ELISpot plates were coated under the same conditions with sporozoite lysate or with recombinant CSP (as positive control) or 
BSA (negative control). To determine the impact of the sporozoite preparation, SPZ were prepared by two different methods: (1) crude preparation using the Ozaki method [16]; (2) clean preparation by dissecting salivary glands from the mosquito heads. For the ease of performing the experiments and based on previous data obtained from the sporozoite-based ELISA, SPZ were isolated using both preparation techniques and then frozen for later use. PBMCs from four CSP-immune donors were plated at a cell concentration of $5 \times 10^{5}$ cells/well and tested for reactivity (Fig. 1). The clean dissection provided stronger signals for all four donors. Therefore, all subsequent experiments were conducted using SPZ from cleanly dissected salivary glands. It is not clear why the crude sporozoite preparation yielded lower responses, but one possible explanation is that debris interferes with the reactivity of the antibodies.

\section{Optimization of assay sensitivity}

Initial experiments revealed that the signal strength was significantly dependent on the vaccine or malariaexposure of the donors. To determine the detection threshold of the assay and to also increase the sensitivity of the assay, the following two experiments were conducted: (1) titre the cell number of CSP-immune PBMC to determine the dose response and detection threshold (Fig. 2); (2) compare the signal strength when using either CSP-immune PBMC or CSP-immune B cells in the assay (Fig. 3).

Titrating PBMC revealed that a linear response was achieved with cell numbers of between $10^{5}$ to

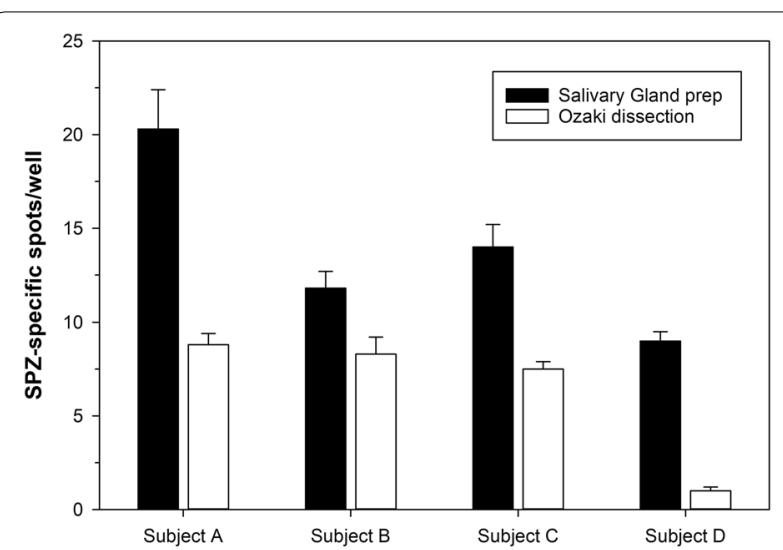

Fig. 1 Impact of sporozoite preparation on signal strength. ELISpot plates were coated with lysates of sporozoite (30,000/well) either dissected using the Ozaki method (white bars) or by preparing salivary glands (black bars). $5 \times 10^{5} \mathrm{PBMC} /$ well from four different CSP-immune donors were plated. Data are expressed as the average number of sporozoite-reactive B cells (error bar represents standard deviation of triplicate wells)

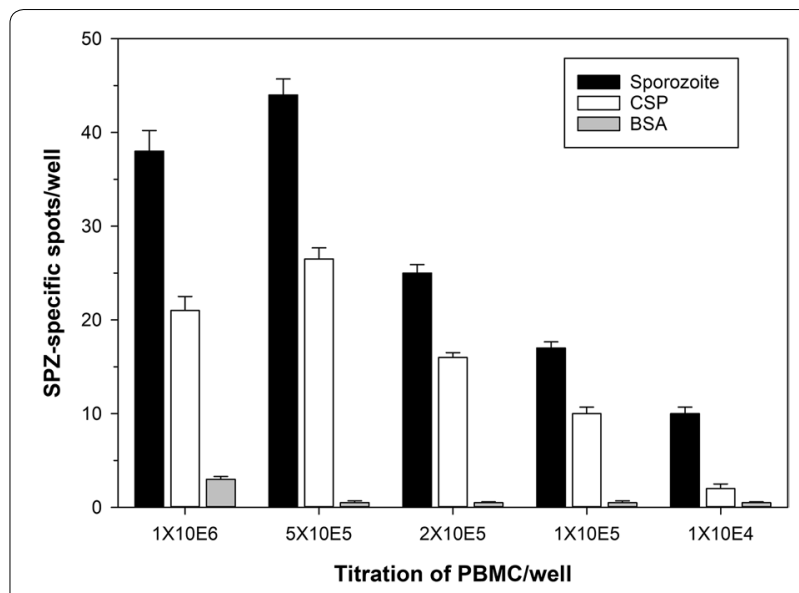

Fig. 2 CSP-immune PBMC react with sporozoite lysates in an ELISpot format. Wells were either coated with sporozoite lysate (30,000/well, salivary gland preparation) or recombinant CSP protein (positive control) or BSA (negative control) and titrations of CSP-immune PBMC (indicated on $x$-axis) plated to determine the optimal cell concentration. Data (representative experiment) are expressed as the average number of reactive B cells (error represents SD of triplicate wells)

$5 \times 10^{5}$ cells/well. Adding more cells $\left(10^{6}\right.$ cells/well $)$ did not increase signal strength. In contrast, plating a higher cell number than $5 \times 10^{5}$ cells/well is not recommended by the manufacturer. A range of $2 \times 10^{5}$ to $5 \times 10^{5}$ is optimal and provides flexibility in cases where the sample volume is limited.

Human peripheral blood consists of $1 \%$ to $7 \%$ B cells. This raises the question whether purifying $B$ cells with magnetically labelled CD19 could increase the sensitivity of the assay because it would be equivalent to plating a larger number of PBMCs (plating $10^{5} \mathrm{~B}$ cells would equate to $1.4-10 \times 10^{6} \mathrm{PBMC}$ ), which is not possible due to crowding of cells in the well. Comparative experiments were set up whereby PBMCs versus positively enriched B cells (using CD19 microbeads) from each donor were tested for reactivity in the sporozoitebased ELISpot. Three volunteers from a previously conducted malaria clinical trial (RTS,S vaccine) were tested to assess parasite responses for pre-immune, post-3 (pre-challenge; 2 weeks after the last immunization) and post challenge (4 weeks after controlled human malaria infection) time points. Purified B cells showed higher spots per well as compared to unfractionated PBMCs (Fig. 3). The results demonstrated a statistically significant increase in the signal between purified $\mathrm{B}$ cells and PBMCs. Therefore, subsequent assays were always conducted with purified B cells after the polyclonal stimulation of PBMCs. 


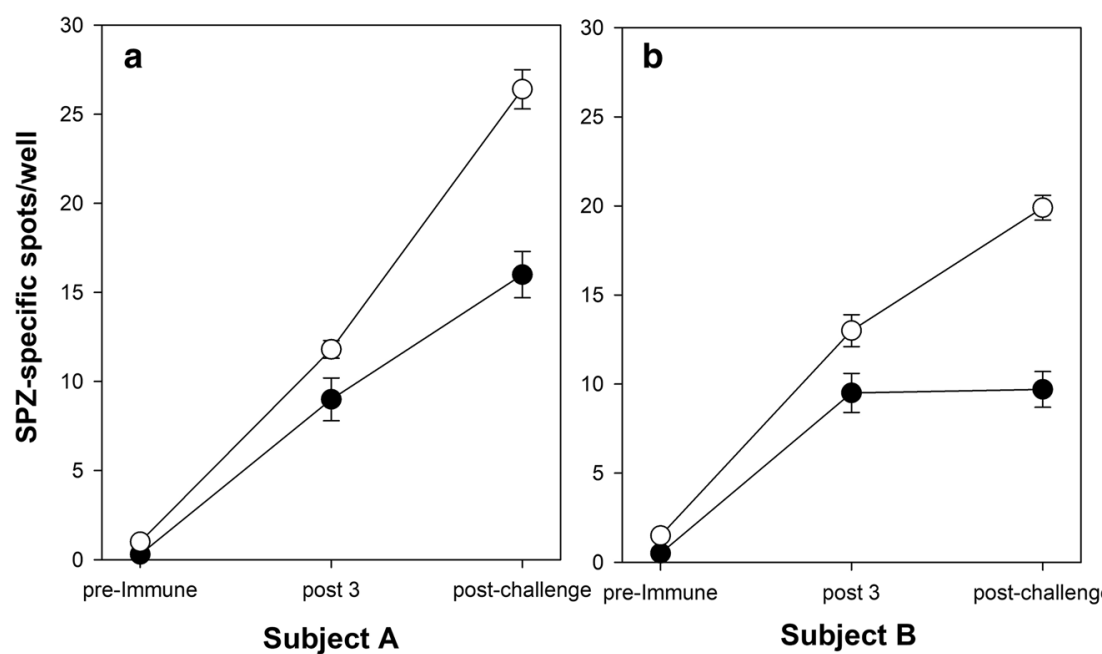

Subject A
Subject B

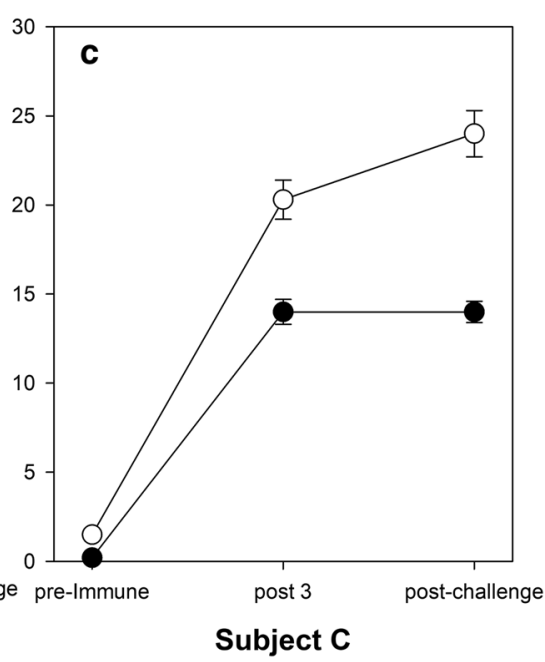

PBMC

Fig. 3 Enrichment of B cells prior to plating onto the sporozoite-ELISpot improves the assay sensitivity. Three CSP-immune donors (a, $\mathbf{b}$, and $\mathbf{c}$ representing the donors) were tested to measure the parasite response for pre-immune, post 3 (i.e., pre challenge), and post challenge (4 weeks after controlled human malaria infection) time points. $5 \times 10^{5} \mathrm{PBMC} /$ well (black symbol) or $10^{5}$ purified B cells/well (white symbol) were plated. Data expressed as mean (SD) of triplicate wells

\section{Detection of parasite-specific, IgM and lgG-producing B cells}

The next step after establishing the primary conditions for the SPZ-based ELISpot assay was to determine the frequency of IgM and IgG producing B cells that recognize epitopes on $P$. falciparum SPZ. Pre-immune, post 3 (i.e., pre-challenge; 2 weeks post last immunization), and post challenge (4 weeks post controlled human malaria infection) time points from two different CSP-immune donors were tested for reactivity in the SPZ-based ELISpot. After the overnight incubation of purified B cells in the ELISpot plates, cells were removed and the plates either probed with biotinylated anti-human IgM or anti-human IgG monoclonal antibodies followed by Streptavidin-AP to assess the frequency of CSP-specific IgM or IgG producing $B$ cells (Fig. 4). The results demonstrate that vaccination results in significant increases in the number of SPZ-specific IgM and IgG-producing B cells. A weak response was observed in pre-immune samples; this could reflect cross-reactivities of pre-existing antibodies to other pathogens such as Toxoplasma. Similarly, there is drop in the frequency of SPZ-specific B cells after a controlled human malaria infection (challenge). This could be due to the sequestration of SPZ-specific $B$ cells in immunological organs and warrants further analysis.

\section{Application of sporozoite-specific B cell ELISpot protocol} The next step was to apply the SPZ-based ELISpot assay to human vaccine models by testing pre-immune and pre-challenge PBMCs from a RAS clinical trial under the established conditions $\left(10^{5}\right.$ purified B cells/well). Results were stratified based on the protective status of the donors $(n=8$ for protected subjects and $n=4$ for not-protected subjects); in the IMRAS trial, donors were immunized by receiving approx. 960 infectious bites of P. falciparum infected, irradiated An. stephensi mosquitoes and protective efficacy assessed in a controlled human malaria infection (CHMI). In the CHMI study, participants are challenged with five bites of $P$. falciparum-infected mosquitoes and evaluated daily for the presence of blood-stage parasites. Only donors that remain parasite-free after 28 days are considered protected (i.e., sterile protection). IgG responses were measured against SPZ and CSP (Fig. 5). The results provided several important observations: (a) the frequency of CSPspecific B cells is significantly lower compared to B cells reactive to SPZ. While the data confirm that CSP is one of the major antigens recognized by malaria-immune $\mathrm{B}$ cells, they also show that other sporozoite-specific antigens are present and recognized by a significantly higher number of B cells; (b) the frequency of CSP-specific B cells is significantly higher in subjects protected by the RAS vaccine ( $p=0.016,2$-sided T-test); (c) the frequency 

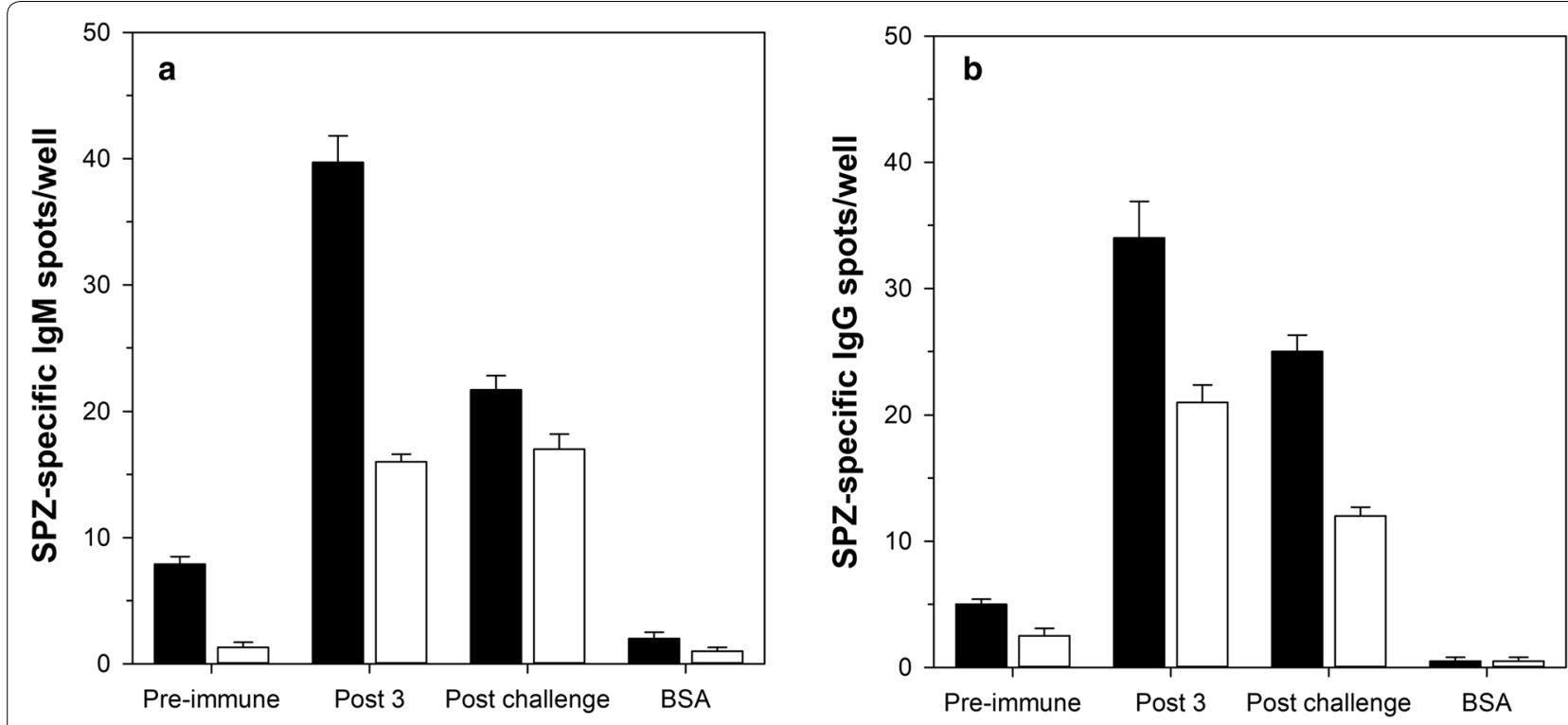

Fig. 4 Detection of parasite-specific, IgM- and lgG-producing B cells. Parasite-specific IgM response (a). Parasite-specific lgG response (b). Data are represented as the mean number of sporozoite-specific spots per $10^{5}$ B cells (triplicate wells). Time points tested: pre-immune, post 3 (i.e., pre-challenge), post challenge (4 weeks after controlled malaria infection). BSA-coated (BSA) wells demonstrate non-specific binding of antibodies to the plate. Fill of bars indicates donors $(n=2)$
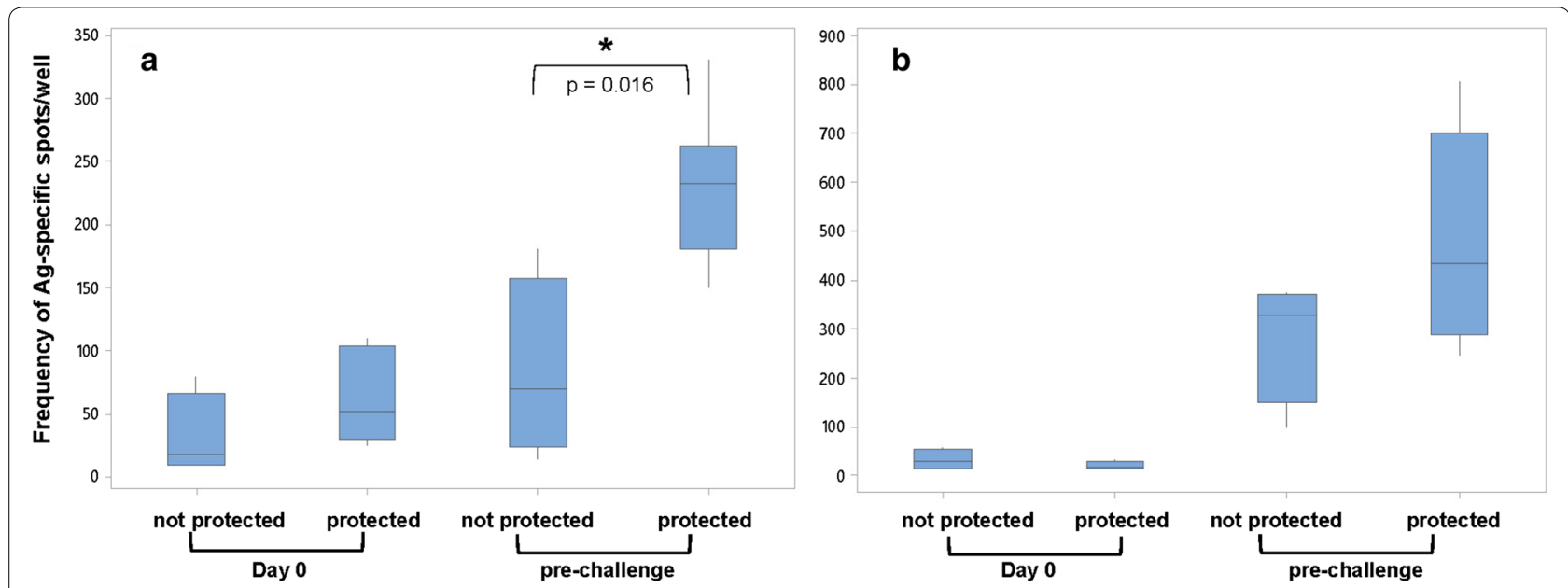

Fig. 5 Sporozoite-ELISpot can discern between protected and non-protected RAS vaccinated subjects. Box plots represent the frequency of CSP-specific (a) or SPZ-specific (b) IgG-spots per $10^{5}$ plated B cells from protected $(n=8)$ and non-protected $(n=4)$ subjects per time point. Time points: pre-immune and pre-challenge (i.e., 2 weeks after last immunization, day of challenge)

of sporozoite-specific $\mathrm{B}$ cells has a strong trend to be higher in protected subjects $(\mathrm{p}=0.08,2$-sided T-test). The latter observation suggests the utility of this readout for identifying immune correlates of protection. The role of CSP-specific antibodies, and therefore B cells, in mediating sterile protection has long been recognized [11, 15, $19,20]$. Not all antigens recognized by IMRAS-induced antibodies contribute to protection. The weaker correlation between frequency of sporozoite-specific B cells and protection (Fig. 5b) would suggest that some of the recognized antigens either do not correlate with protection or may even correlate with susceptibility.

\section{Conclusion}

The successfully developed B-cell ELISpot assay measures the frequency of parasite-specific $B$ cells. This assay is capable of detecting malaria-specific B cells secreting sporozoite-specific IgM and IgG in vitro. 
The newly developed assay shows high sensitivity and is robust enough to detect $B$ cell responses induced by two distinct vaccine platforms in humans. This new immunoassay can assist in the evaluation of vaccine induced B-cell responses based on native sporozoite antigens and support the identification of immune correlates of protection against malaria infections.

\begin{abstract}
Abbreviations
BSA: bovine serum albumin; CSP: circumsporozoite protein; FBS: fetal bovine serum; GAS: genetically engineered sporozoites; HBsAg: hepatitis B surface antigen; IMRAS: immunization by mosquito bite with radiation attenuated sporozoites; mAb: monoclonal antibody; NEAA: non-essential amino acids; PBMC: peripheral blood mononuclear cells; RAS: radiation attenuated sporozoites; SPZ: sporozoite; WPV: whole parasite vaccines.
\end{abstract}

\section{Acknowledgements}

The authors would like to thank Mr. Kelly Palmer and Ms. Elizabeth Duncan for the mosquito dissections. The authors greatly appreciate the support of Drs. William Zonta, Philippe Moris, Michel Janssens, and Erik Jongert by reviewing the manuscript.

\section{Declarations}

GlaxoSmithKline is the inventor of the RTS,S/AS01 vaccine and conducted the MAL-27 clinical trial in collaboration with WRAIR. JEE is a military service member, ESB-L is a government employee. Title 17 U.S.C. $\$ 105$ provides that "Copyright protection under this title is not available for any work of the United States Government, but the United States Government". Title 17 U.S.C. $\S 101$ defines US Government work as "work prepared by a military service member or employee of the US Government as part of that person's official duties".

\section{Disclaimer}

Material has been reviewed by the Walter Reed Army Institute of Research. There is no objection to its presentation and/or publication. This paper has been approved for public release with unlimited distribution. The opinions or assertions contained herein are the private views of the authors, and are not to be construed as official, or as reflecting the views of the Department of the Army or the Department of the Navy or the Department of Defense nor the U.S. Government. The investigators have adhered to the policies for protection of human subjects as prescribed in AR 70-25.

\section{Authors' contributions}

TA performed the experiments. TS, TMR, SD, and JEE provided crucial reagents for the assay and edited the manuscript. ESB-L and TA designed the experiments and compiled the manuscript. All authors read and approved the final manuscript.

\section{Funding}

This work was supported by the US Military Infectious Disease Research Project and the US Army Medical Research and Materiel Command. The IMRAS Trial was supported by Grant OPP1034596 from the Bill \& Melinda Gates Foundation.

\section{Availability of data and materials}

The data and detailed protocol can be made available upon request from the corresponding author.

\section{Ethics approval and consent to participate}

The IMRAS study protocol was approved by the NMRC IRB, the MAL27 study protocol was approved by the WRAIR IRB in compliance with all applicable federal regulations governing the protection of human subjects. The MAL27 sample use was reviewed by the WRAIR Human Subjects' Protection Branch which determined that the research does not involve human subjects (NHSR protocol WRAIR\#2142) as the samples used were de-identified and no link between samples and subjects exists.
Consent for publication

Not applicable.

\section{Competing interests}

The authors declare that they have no competing interests.

\section{Author details}

${ }^{1}$ Malaria Vaccine Branch, US Military Malaria Research Program, Walter Reed Army Institute of Research, Silver Spring, MD, USA. ${ }^{2}$ Division of Entomology, Walter Reed Army Institute of Research, Silver Spring, MD, USA. ${ }^{3}$ Malaria Department, Naval Medical Research Center, Silver Spring, MD, USA.

Received: 1 April 2019 Accepted: 18 May 2019

Published online: 29 May 2019

\section{References}

1. Crompton PD, Moebius J, Portugal S, Waisberg M, Hart G, Garver LS, et al. Malaria immunity in man and mosquito: insights into unsolved mysteries of a deadly infectious disease. Ann Rev Immunol. 2014;32:157-87.

2. Menard R, Tavares J, Cockburn I, Markus M, Zavala F, Amino R. Looking under the skin: the first steps in malarial infection and immunity. Nat Rev Microbiol. 2013;11:701-12.

3. Lindner SE, Swearingen KE, Harupa A, Vaughan AM, Sinnis P, Moritz RL, et al. Total and putative surface proteomics of malaria parasite salivary gland sporozoites. Mol Cell Proteomics. 2013;12:1127-43.

4. Regules JA, Cicatelli SB, Bennett JW, Paolino KM, Twomey PS, Moon JE, et al. Fractional third and fourth dose of RTS,S/AS01 malaria candidate vaccine: a phase 2 a controlled human malaria parasite infection and immunogenicity study. J Infect Dis. 2016;214:762-71.

5. Stoute JA, Kester KE, Krzych U, Wellde BT, Hall T, White K, et al. Long-term efficacy and immune responses following immunization with the RTS,S malaria vaccine. J Infect Dis. 1998;178:1139-44.

6. Bijker EM, Borrmann S, Kappe SH, Mordmuller B, Sack BK, Khan SM. Novel approaches to whole sporozoite vaccination against malaria. Vaccine. 2015:33:7462-8.

7. Epstein JE, Paolino KM, Richie TL, Sedegah M, Singer A, Ruben AJ, et al. Protection against Plasmodium falciparum malaria by PfSPZ vaccine. JCI Insight. 2017;2:e89154.

8. Vaughan AM, Kappe SHI. Genetically attenuated malaria parasites as vaccines. Exp Rev Vaccines. 2017;16:765-7.

9. Rieckmann KH, Beaudoin RL, Cassells JS, Sell KW. Use of attenuated sporozoites in the immunization of human volunteers against falciparum malaria. Bull World Health Organ. 1979;57(Suppl 1):261-5.

10. Ishizuka AS, Lyke KE, DeZure A, Berry AA, Richie TL, Mendoza FH, et al. Protection against malaria at 1 year and immune correlates following PfSPZ vaccination. Nat Med. 2016;22:614-23.

11. Knepper TA, Duncan EH, Savransky T, Bergmann-Leitner ES. Novel ELISA method as exploratory tool to assess immunity induced by radiated attenuated sporozoites to decipher protective immunity. Malar J. 2017;16:484.

12. Langhorne J, Cross C, Seixas E, Li C, von der Weid T. A role for B cells in the development of $\mathrm{T}$ cell helper function in a malaria infection in mice. Proc Natl Acad Sci USA. 1998;95:1730-4.

13. Meding SJ, Langhorne J. CD4+T cells and B cells are necessary for the transfer of protective immunity to Plasmodium chabaudi chabaudi. Eur J Immunol. 1991;21:1433-8.

14. Nahrendorf W, Scholzen A, Bijker EM, Teirlinck AC, Bastiaens GJ, Schats $\mathrm{R}$, et al. Memory B-cell and antibody responses induced by Plasmodium falciparum sporozoite immunization. J Infect Dis. 2014;210:1981-90.

15. Schwenk R, DeBot M, Porter M, Nikki J, Rein L, Spaccapelo R, et al. IgG2 antibodies against a clinical grade Plasmodium falciparum CSP vaccine antigen associate with protection against transgenic sporozoite challenge in mice. PLoS ONE. 2014;9:e111020.

16. Ozaki LS, Gwadz RW, Godson GN. Simple centrifugation method for rapid separation of sporozoites from mosquitoes. J Parasitol. 1984;70:831-3.

17. Coleman J, Juhn J, James AA. Dissection of midgut and salivary glands from Ae. aegypti mosquitoes. J Vis Exp. 2007;5:228.

18. Kester KE, Cummings JF, Ofori-Anyinam O, Ockenhouse CF, Krzych U, Moris $\mathrm{P}$, et al. Randomized, double-blind, phase $2 \mathrm{a}$ trial of falciparum 
malaria vaccines RTS,S/AS01B and RTS,S/AS02A in malaria-naive adults: safety, efficacy, and immunologic associates of protection. J Infect Dis. 2009;200:337-46.

19. Aliprandini E, Tavares J, Panatieri RH, Thiberge S, Yamamoto MM, Silvie O, et al. Cytotoxic anti-circumsporozoite antibodies target malaria sporozoites in the host skin. Nat Microbiol. 2018;3:1224-333.

20. Kurtovic L, Agius PA, Feng G, Drew DR, Ubillos I, Sacarlal J, et al. Induction and decay of functional complement-fixing antibodies by the RTS,S malaria vaccine in children, and a negative impact of malaria exposure. BMC Med. 2019;17:45.

\section{Publisher's Note}

Springer Nature remains neutral with regard to jurisdictional claims in published maps and institutional affiliations.
Ready to submit your research? Choose BMC and benefit from:

- fast, convenient online submission

- thorough peer review by experienced researchers in your field

- rapid publication on acceptance

- support for research data, including large and complex data types

- gold Open Access which fosters wider collaboration and increased citations

- maximum visibility for your research: over $100 \mathrm{M}$ website views per year

At BMC, research is always in progress.

Learn more biomedcentral.com/submissions 